mantle and of mantle lying much closer to the surface in a high-temperature, highpressure apparatus. He then placed these samples together in the same apparatus and heated them for $10 \mathrm{~min}$. After cooling the samples, he separated them and analyzed their mineralogical compositions.

"We generally think of magmas as moving very slowly and geological processes as occurring over hundreds of thousands of years, so it's really amazing how much happens in just 10 minutes," Lundstrom said. "In that brief time, considerable migration occurred as sodium diffused through the melt between mineral grains, significantly altering the composition of the material."

In a positive-feedback loop, the infiltrating sodium causes orthopyroxene to break down into olivine plus the peculiar composition melt. This melt, in turn, causes even more sodium to be pulled in. Lundstrom said that the increase in olivine and the decrease in orthopyroxene within the diffusively infiltrated piece of mantle may explain another perplexing observation: the anomalous ratio of these minerals found in the shallow mantle region beneath mid-ocean ridges.

He said, "We tend to think of volcanic edifices-like the Hawaiian Islands-as resulting from hot spots in the mantle. But these results show that sodium-rich, silica-poor magma can cause the surrounding mantle to melt through a chemical reaction without invoking huge quantities of heat." Lundstrom also said that the diffusion process could be important to melting the cold lithospheric "plates" that cover the earth.

\section{Model of Interactions Between Coupled Atomic Bose Condensate and Molecular Gas Predicts Formation of Molecular Bose Condensates}

Researchers investigating dilute BoseEinstein condensation (BEC) have proposed a novel, non-Arrhenius chemical kinetics at ultralow temperatures. D.J. Heinzen and R.H. Wynar at the University of Texas-Austin, and P.D. Drummond and K.V. Kheruntsyan at the University of Queensland in Australia have named this phenomenon "superchemistry." Defined as "the coherent stimulation of chemical reactions via macroscopic occupation of a quantum state by a bosonic chemical species," superchemistry involves giant collective oscillations between a dilute, trapped Bose-condensed atomic gas and a diatomic molecular Bose gas. Coupling takes place between these two species through coherent Raman transitions, and leads to the formation of a molecular Bose condensate through stimulated emission of molecular bosons.

Of particular interest in their model is the introduction of the particle number nonconserving potential, similar to parameters used to describe nonlinear optics. In the Arrhenius kinetics model the rates of chemical reaction are independent of the number of particles of product species and tend toward zero at ultralow temperatures. However, as published in the May 1 issue of Physical Review Letters, the proposed superchemistry kinetics model shows that chemical conversions inside a Bose condensate are caused by coherent stimulated emission, which is enhanced by the number of molecules already occupying the ground state. The current research considers the specific case of stimulated Raman coupling induced by two laser fields of different frequencies; resonant coupling occurs when Raman detuning goes to zero. A trap potential localizes the interaction volume.

The investigators see potential applications in the area of quantum-controlled chemical synthesis.

TIM PALUCKA

\section{Wavelength-Interchanged Optical Sensor Facilitates Biochemical Analysis}

M. Wiki and R.E. Kunz from Centre Suisse d'Electronique et de Microtechnique in Zurich, Switzerland reported in the April 1 issue of Optics Letters a novel sensor that allows the simultaneous measurement of several different biochemicals. The miniature $(18 \mathrm{~cm} \times 6 \mathrm{~cm} \times 4 \mathrm{~cm})$ integratedoptical sensor relies on wavelengthmodulation techniques to interrogate multiple waveguide sensing regions in a single integrated-optical chip for accurate measurements of effective refractive index changes at a high data rate. The sensor can exploit the wide tuning range of verticalcavity surface-emitting lasers to obtain high-resolution spectral measurements of refractive-indices. The integrated optical chip structure is based upon $\mathrm{TiO}_{2}$ waveguide structures fabricated over a polycarbonate substrate onto which grating structures are fabricated using a hot embossing process. The experiments with this sensor yielded a resolution (in units of the effective refractive index) $\Delta N_{p p}=10^{-7}$. The researchers' method consists of an input grating-coupler scheme. The structure consists of two different grating pads, one used as a reference pad and one of which is obstructed by the transparent biochemical layer to be analyzed. The position of the output resonance peak is a measure of the effective refractive index of the sensor chip, and hence contains the desired information about the biochemical layer, information that can be extracted by applying a theoretical model of the IO sensor chip. Integrated optics is one well-known technique for label-free detection of (bio)electrical reactions, and has been used for many years. Previously developed integrated-optical sensors include sensors based on uniform grating couplers, chirped grating couplers, surface-plasmon resonance, resonant mirrors, and difference Mach-Zehnder and Young interferometers. But for practical applications, most label-free sensors either do not achieve the desired resolution for multiple channels, or are very bulky and expensive.

Claudiu Muntele

\section{Blends of Electron- and Ion- Conducting Polymers Yield Fast Electrodes in Supercapacitors}

A team of researchers at Linköping University in Sweden described the synthesis of polymer blends that are both electron- and ion-conducting in the May issue of Electrochemical and Solid-State Letters. The blends consist of electronically conductive PEDOT-PSS (poly(3,4-ethylenedioxythiophene)-polystyrenesulfonate) and ionically conductive PEO (polyethylene oxide) and give higher energy densities at high power densities compared to pure PEDOT-PSS polymers. The network morphology of the PEDOT-PSS extends these electrodes into the third dimension, making them promising materials for use in supercapacitors, sensors, and electrocatalystsp.

"Electronically conducting polymers are of interest for use as electrode materials because their amorphous nature gives them good permeability to electrolytes," said Olle Inganäs from Linköping's Applied Physics Division. "However, ionic compatibility is often low, which limits the charge transfer in these systems by the slow ionic exchange." In recent years, the researchers obtained a patent on a process that involves doping PEDOT with PSS and ionically crosslinking the resulting polymer into highly swollen hydrogels. This yields 3D networks of electronically conducting polymers with more than $90 \%$ in an aqueous phase that provides good ionic mobility. "But application of PEDOT-PSS hydrogel electrodes is restricted to aqueous systems, which have comparatively small windows for electrochemical potentials", said Soumyadeb Ghosh, a postdoc at Linköping University. In order 